\title{
José Manuel Zozaya y el inicio de las relaciones de México con EU
}

\author{
Ana Rosa Suárez Argüello \\ INSTITUTO MORA
}

\begin{abstract}
A través de la correspondencia del primer ministro plenipotenciario de México en Estados Unidos, se recrea, la imagen que de este país y sus habitantes tuvo la diplomacia mexicana.
\end{abstract}

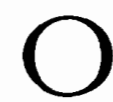

riginario de Guanajuato, José Manuel Zozaya y Bermúdez nació en 1775, en las postrimerías del periodo colonial. Estudiante del Colegio de San Ildefonso, se convirtió en abo- gado, llegando a pertenecer al Colegio de Abogados de México desde 1805. En la Audiencia de México fungió como diputado, examinador y rector de letrados.

Partidario de Iturbide, Agustín I lo nombró consejero honorario de Estado al proclamarse el imperio. A sugerencia imperial, José Manuel de Herrera, secretario de Relaciones Interiores y Exteriores, lo designó enviado extraordinario y ministro plenipotenciario en Washington, recibiendo el nombramiento oficial el 25 de septiembre de 1822. En Estados
Unidos lo sorprendió la caída de Iturbide regresando a su país en mayo de 1823 , luego de dejar acreditado como encargado de negocios ad interim al coronel José Antonio Torrens, secretario de la legación.

Al parecer, el ex ministro quedó fuera de la política y durante algún tiempo se dedicó a administrar la fábrica de papel que estableció en el molino de Loreto, en el pueblo de Tizapán, a fines de 1823 o principios de 1824, con artesanos y maquinaria traídos de Estados Unidos. Con papel de esta fábrica se imprimiría la segunda edición de la Constitución de 1824. Posteriormente representaría al estado de Guanajuato en la Cámara de Diputados del Tercer Congreso General.
1 
Faltan noticias sobre nuestro personaje en años posteriores. No obstante, se sabe que murió en la ciudad de México en 1853.

Como primer enviado extraordinario y ministro plenipotenciario del México independiente en Estados Unidos, su misión en Washington constituyó un fracaso. Preludió así la historia de una relación que no siempre ha sido la mejor.

Las instrucciones generalesy reservadas de Zozaya eran solicitar el reconocimiento de la independencia y del imperio mexicano; celebrar tratados de amistad, alian$\mathrm{za}$, comercio, arreglo de límites, etc.; procurar el auxilio del gobierno y los particulares norteamericanos en caso de reanudarse la guerra con España; negociar un préstamo de 10000000 de pesos, pudiendo para ello hipotecar las rentas imperiales en general ocualquiera de ellas en particular; averiguar qué aranceles mexicanos eran gravosos para Estados Unidos, $y$, finalmente, enterarse de la verdadera opinión de los "republicanos" de aquel país sobre la forma de gobierno y la dinastía elegidas en México e informarse de sus intenciones sobre la extensión de los límites de Luisiana y las Floridas.

${ }^{1}$ Para algunos datos sobre José Manuel Zozaya, véase Diccionario Porría de bistoria, biografia y geografía de México, 2a. ed., Porrúa, México, 1964, p. 1760 ; La diplomacia mexicana, intr. de Federico Gamboa, Secretaría de Relaciones Exteriores, México, 1910, t. I, pp. 75-112; Hans Lenz, Loreto, Historia y euolución de una fábrica de papel, Fá-
Zozaya presentó cartas credenciales al presidente James Monroe el 12 de diciembre de 1822, fecha oficial del reconocimiento de la independencia de México y del imperio de Iturbide por parte de Washington. Pese a la cordial acogida que se le brindó, el diplomático mexicano se percató rápidamente de las ambiciones de los norteamericanos. Por eso prefirió no plantearles el asunto de los límites, si bien era conveniente que ratificaran los trazados en el Tratado OnísAdams y, con base en argumentos que a la fecha se escuchan, se negó a discutir un tratado de comercio, seguro de que, más fuertes, los norteamericanos se llevarían todas las ventajas. ${ }^{1}$

De modo que Zozaya no emprendió ninguna negociación. Su misión se limitaría a comunicar a sus superiores algunas de sus impresiones sobre Estados Unidos y los estadunidenses. Las cartas que se presentan a continuación son las primeras que envió a México después del reconocimiento del imperio. ${ }^{2}$ Vale destacar en ellas las opiniones de quien fue el primer viajero-diplomático mexicano en Estados Unidos.

bricas de Papel Loreto y Peña Pobre, México, 1956, pp. 47-61; Luis G. Zorrilla, Historia de las relaciones entre México y los Estados Unidos de América, 1800-1958, 2a. ed., 2 vols., Editorial Porrúa, México, t. 1. pp. 53-55 (Biblioteca Porrúa, 29-30).

${ }^{2}$ Se modernizó la ortografia, se desataron las abreviaturas y se anotaron las cartas seleccionadas. 
Excelentísimo señor:

Aprovecho la ocasión de escribir a vuestra excelencia por conducto del conde Lucchesi, que se halla hoy en Nueva York, a quien he franqueado pasaporte para pasar a ese imperio a tratar asuntos del duque de Monteleón.

Es mi objeto ahora no perder esta coyuntura que se presenta para comunicar a vuestra excelencia de oficio, que a pesar de las diligencias públicas y secretas de algunos enemigos de nuestro país para prevenir la opinión del gobiemo de estos Estados contra la forma del nuestro y contra mí como su representante; he sido presentadoel día 12 del comiente por el secretariode Estado ${ }^{3}$ al presidente de estos Estados, por quien fui recibido con la misma etiqueta y ceremonial que se reciben los demás ministros de las demás potencias, y habiéndole entregado mis credencialesmecontestóquedarreconocidoyadmitidocomoministropúblicoyenviado extraordinario plenipotenciario, lo mismo que los demás ministros extranjeros residentes en este país.

Precedió a esto la entrega en copia de mis credenciales al ministro de Estado para que previamente se examinaran y, al día siguiente de mi presentación y reconocimiento, se anunció de oficio en el papel ministerial intitulado National Intelligencer de que remito a vuestra excelencia un ejemplar.

Tengo entendido que el ministro de España pasó una nota a este gobiemo protestando contra el reconocimiento de nuestra independencia y contra el acto de mi presentación en esta capital si se me admitía con el carácter de plenipotenciario de México. No he podido adquirir un dato cierto de este hecho, pero sí es verdad que él residía en Filadelfia, y cuandoyofui a esa ciudad, pasóél pocos días antesaNueva York, en dondehasta hoy permanecesinhaberse presentadoenesta capital, siendo él solo el único ministro extranjero que está hoy fuera de la corte.

De todo haré a vuestra excelencia una explicación más expresiva para remitir cuandovayan dos denuestras goletascañoneras, que debensalirmuy prontopara Alvarado.

Dios guarde a vuestra excelencia muchos años. Washington, 20 de diciembre de 1822. Zozaya. Excelentísimo señor secretario de Estado y del Despacho de Relaciones Interiores y Exteriores del imperio. ${ }^{4}$

\footnotetext{
${ }^{3}$ John Quincy Adams era secretario de Estado.
}

${ }^{4}$ Secretaría de Relaciones Exteriores (SRE), Archivo de la Embajada de México en Estados Unidos (AEMEU), t. 424, núm. 4, ff. 7-8. Parte de la correspondencia de Zozaya aparece en Diplomacia, 1910, t. I, pp. 75-112. 
Excelentísimo señor:

Con fecha veinte de este mes escribí a vuestra excelencia, por conducto de Nueva York, el principal de que va ahora duplicado, ofreciendo hacerloextensamenteen esta ocasión que van dos de nuestras goletas; y en efecto lo hago por conducto de don Ricardo Meade, de quien por residir en Filadelfia que es puerto, tendré necesidad de valerme siempre. ${ }^{5}$

Conforme a mis instrucciones y a la costumbre que aquí se observa anuncié mi llegada el día 1 del corriente al secretario de Estado acompañándole copia de miscredenciales; yéstemeenvióel billetequeacompañooriginal para quenuestra etiqueta se arregle a la suya. En virtud de la citación que en él me hace concurricon el secretario en el departamento, no en su casa, y después de los cumplidos corrientes manifestó extrañar la falta de algún documento o carta dirigida para el presidenteo para el gobiemo, a más dela credencial que había manifestado; a que lesatisfice diciéndolequeelfin principal de mivenidacercadeestegobiemo, según manifestaba mi credencial, era procurar el reconocimiento de la independencia bajolaactual forma de gobiernoque tenemos yque, hasta que estonoseverificase, nopodiaelemperadorocabezadenuestrogobiemoentendersedirectamentecon éste, protestándole que éste y no otro había sido el motivo de que el emperador no hubiese escrito según se acostumbra por otros soberanos en iguales casos y ofreciéndole que no obstante esto había cualquiera falta, se cubriría luego que yo escribiera a México. Con esto se dio por satisfecho ofreciéndome que no habría dificultad en admitírseme con el carácter que traía de México, y me citó para la una del día siguiente a fin de ser introducido y presentado al presidente. Fui a la hora convenida, y sinla máspequeñademoramerecibióelsecretario, einmediatamente me hizo pasar a la pieza siguiente en la que estaba ya el presidente, y habiéndole entregadomiscredenciales originales y felicitándolo a nombre delemperador, me contestó con urbanidad concluyendo con que quedaba admitido como ministro plenipotenciariodeMéxicolomismoquelosdemásdelasotrasnaciones. Después me pasóel secretariola minuta queoriginal acompañoconsu traducción, para que me sirviese de gobiemo, según me dijo de palabra, en la etiqueta que debía observar, la que no vino acompañada con oficio.

Lacostumbreaquíesquelosministros visitanalossecretariosy senadoresnada más: los representantes en el Congreso, los ministros extranjeros y cualesquiera otras personas que quieran tratar al ministro que llega de nuevo lo visitan, sin que éste anticipe ningún aviso ni haga cumplido alguno. Así lo he practicado yo y he

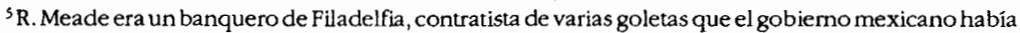
mandado construir para bloquear la fortaleza de San Juan de Ulúa. 
sido correspondido de los secretarios y senadores y visitado de muchos representantes del Congreso y de otras personas caracterizadas, menos los ministros extranjeros que ninguno de ellos ni de sus respectivas legaciones me ha visitado.

Para el día 24 de éste hizoel presidente un convite en mi obsequio para el que me convidó con el tiquet [sid que acompaño. Fueron 40 los de mesa, entre los cualesestaba el encargadode Suecia y el de Francia, el cónsul general de Inglaterra y dos de la misma legación, pero noel ministro inglés; y hasta ahora no he podido cerciorarme si su falta fue por no concurrir conmigo para no comprometerse con su corte que no ha reconocido nuestra independencia según algunos me han asegurado, o si se excusó como otros me han informado, o el presidente no lo convidó para evitar compromisos sobre la preferencia del asiento que se me había deconcedera míporserelconviteen miobsequio. Para mañana me ha convidado a la mesa el ministro de Estado; también es convite de etiqueta y entiendo que ha invitadoigualmentealministroinglésyquehaadmitido.Supongoquese manejará con absoluta indiferencia conmigo lo mismo que hicieron los encargados de negocios que asistieron a la mesa del presidente. Para pasado mañana 27 tengo igual convite del ministro de Hacienda: estos son los actos exteriores con que estas personas públicas se explican con los ministros extranjeros a su llegada a la capital, y desde que son reconocidos como tales.

El secretario me pidió verbalmente noticia de todos los que componen la legación, incluyéndose hasta los criados, para que hubiera una noticia en el Departamento de Estado y se les tuviera la consideración debida conforme al derecho establecido entre las naciones.

Al dia siguiente de mi presentación al presidente, se anunció ésta en el papel intitulado National Intelligencer, que es en el que se ponen todas las cosas del gobierno, y se repitió la misma noticia en la Gaceta de Washington.

En la Guía de Forasteros de Washington o Directorio que remito a vuestra excelencia se da también noticia de la legación mexicana después de la de Inglaterra, y en nada absolutamente se me ha faltado de cuantas etiquetas y comedimientos se gastan con los ministros de las demás potencias.

Sólo resta que la embajada se conresponda por este gobiemo y he llegado a entender que esto tendrá efecto dentro de dos semanas y que probablemente recaerá el nombramiento de ministro en mister Hugh Nelson, diputado del Congreso por Virginia, de quien tengo buenos informes y ya me rectificaré ellos. Sospechoque acasoestegobiemoaguarda la llegada de Méxicode misterPoinsett para arreglar sus instrucciones a los informes que éste les dé.

Con esto han quedado burlados los agoreros de México que me presagiaban muymalreciboaquí,ylomismolosescritoressuscorrespondientesoparcialesque 


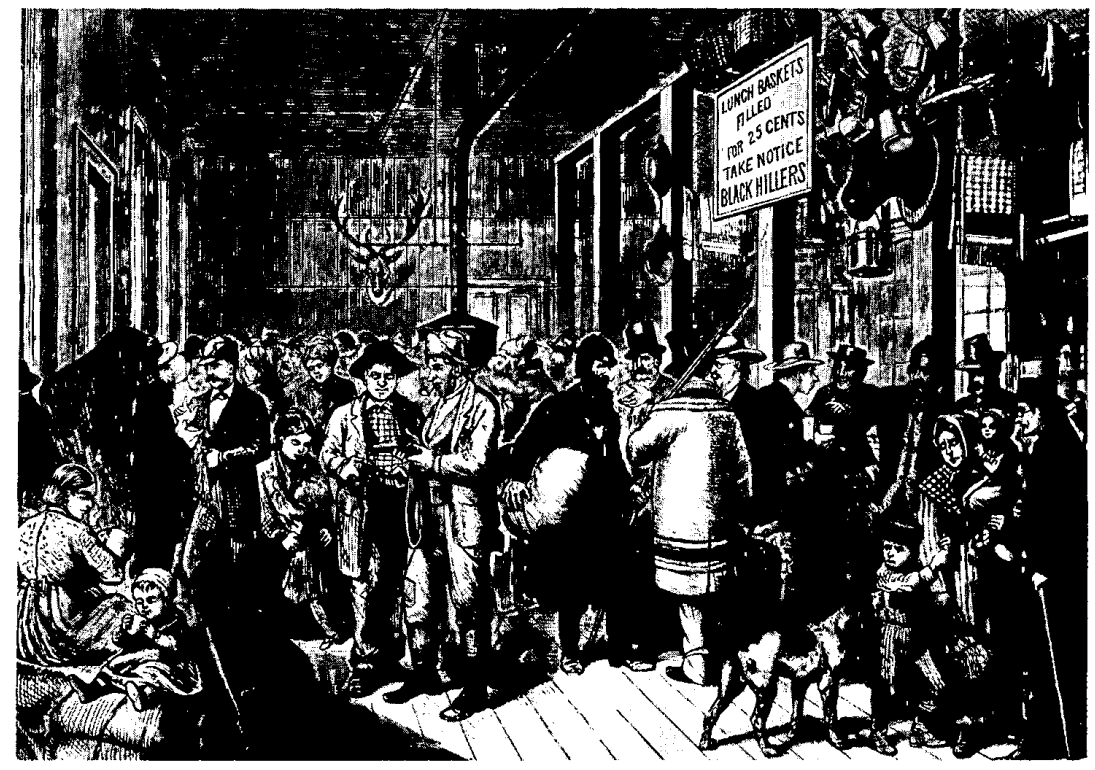

creyeron prevenir la opinión de este gobiemo escribiendo los dicterios de que vuestra excelencia se impondrá por los impresos que le acompaño con sus respectivas traducciones: y tengo la satisfacción de asegurar a vuestra excelencia que ha sidocasi general el despreciocon que se han visto tales papeluchos de que he recibido muy repetidas pruebas.

Dije antes que ofrecial secretario de Estado que se cubriría la falta de no haber escrito el emperador directamente a este gobiemo; y al efecto traté de indagar en eldepartamentosiseacordaba previamentela etiqueta conquesehabía deescribir y meinstruyeron que nose hace sobreesoacuerdoalguno, sinoque los soberanos o jefes de las naciones escriben según les parece, y para arreglar el modo con que nuestro emperador debía hacerlo, pedí se me franquearan algunas credenciales del rey de España, y habiéndome enseñado todo lo conducente a este particular de orden del secretario, saqué el modelo del de España y del de Inglaterra de que acompañocopia. Ahora está en arbitriode su majestad imperial elegir el tratamiento que mejor le acomode. Advierto que el oficial mayor del Departamento de Estado y otros oficiales me dijeron que las cartas debían dirigirse sóloal presidente, y noa los Estados, que las que habíamos visto estaban mal concebidas; y en efecto el Belfield dice que la carta debe ser de jefe a jefe, pero en el departamento no he 
visto ninguna en esta forma y todas hablan con los Estados. Me parece que no se deje de escribir para cubrir mi compromiso y alejar de estos republicanos la idea que acaso podrían concebir de que el emperador no se dignaba entrar en contestaciones directas con su gobiemo. La carta ya no puede ser una credencial, como vienen las de su clase, porque ya sin este requisito estoy reconocido y recibido como ministro; y así creo yo que puede concebirse diciendo su alteza imperial que por su enviado ha sabidoel buen recibimiento que ha tenido: que da gracias por ello y que pide que en lo sucesivo se le seguirá teniendo la misma consideracióntantoaél, comoa su secretarioenelcasoqueporalgúnmotivotenga que dejarlo de encargado de negocios y que a ambos en su respectivo caso se le dé entera fe en todo lo que aseguren a nombre de su majestad imperial, quien por su parte hará lo mismo.

Es absolutamente necesaria esta cláusula de encargado, lo primero porque después del 4 de marzo es inútil la permanencia del ministro en estos Estados; y lo segundo por el extraordinario gasto que demanda su carácter y mucho más no habiendo otro ministro cerca de este gobiemo que el inglés, que gasta un lujo excesivo y él sirve de extremo de comparación. Yo creo que por eso las demás naciones han adoptado el partido de nombrar encargados quienes, aunque son personas bastante autorizadas por sólola denominación, ya nose hallanen el caso de mantenerse en el mismo pie que un ministro. Y a la verdad que yo tengo por mucha bobera el hacer estos gastos y sacrificios que a nada conducen, y los que no puede evitar un ministro, aunque vaya a vivir o a pasearse, que es lo mismo, a cualquier lugar de estos Estados porque alli siguen los convites como me sucedió a mí en Baltimore y Filadelfia.

Dios guarde a vuestra excelencia muchos años. Washington, 20 de diciembre de 1822. Zozaya. Excelentísimo señor secretario de Estado y del Despacho de Relaciones Interiores y Exteriores del imperio. ${ }^{6}$

Excelentísimo señor:

Verificado el reconocimiento de la independencia del imperio mexicano y mi recepciónenclasedeenviadoextraordinarioyministroplenipotenciarionombrado por su majestad el emperador, de que doy cuenta a vuestra excelencia en nota número 5 es de mi deber darla igualmente a vuestra excelencia de los demás encargos que me hizo y de mis demás operaciones contraídas a llenar los diversos objetos de mi comisión.

Ia carta para el ministro de Estado de la república de Colombia que vuestra

${ }^{6}$ SRE, AEMEU, t. 424 , núm. 5 , ff. 8-10v. 
excelencia encargó a mi cuidado para dirigirla, lo he verificado ya por medio de don Ricardo Meade, encargado particular de los negocios del señor Torres, ministro nombrado por aquella república para estos Estados, quien falleció.

A la carta de don Guillermo Duane de igual dirección, por hallarse ahora este individuo residiendo en Colombia [sid y desde luego nos ha hecho mucho falta paralosobjetosquevuestra excelenciameencargó,nohabiendoencontradohasta ahora un sujeto capaz de reemplazarlo por poseer muy poco y con imperfección el idioma español.

El encargado del Brasil, con quien vuestra excelencia me encargó me condujere con la mayor armonía por su buena disposición a entrar en relaciones con el imperio reside en Filadelfia y no es representante por el gobiemo del Brasil, sino un encargado nombrado por una junta en Pernambuco que se ha agregado al Brasil, lo que creo necesario poner en conocimiento de vuestra excelencia.

La imprenta y la fundición que igualmente me encargó vuestra excelencia, estoy informado de que es cosa muy fácil de conseguir en Filadelfia y remitir en la hora que se quiera, y así lo haré luego que vengan fondos del imperio tanto para este objeto como para los demás gastos extraordinarios.

Hasta ahora no heentradoen contestacionesalgunasdirecta ni indirectamente paraentablarrelaciones delimperioconestosEstados, y creoque sobreel particular me debo manejar pasivamente, porque un tratado de alianza en caso que la necesitemos es incompatible con el gobiemo y leyes de estos Estados y más que todo con sus costumbres y modo de pensar. Un tratado de comercio no creo que estemos todavía en el caso de procurarlo, porque la preponderancia de estos Estadossobre nosotros porsu marinay porotrasconsideracionespolíticas, aunque sean de apreciación, les daría un derecho en su concepto para exigir ventajas sin sacar el imperio ninguna a su favor. Creo que esto debe ser obra del tiempo, de la calma y de la reflexión.

Sobrelímites, queesel puntomásinteresante por razón devecindady de miras que acaso puede haber, nada puede hacerse por vía de tratados, supuesto que existe el último con España, que debemos respetar y con el que creo que este gobierno se conforma muy bien. Este punto está más bien sujeto a operaciones materiales para fijar los términos divisorios conforme lo tratado, que a negociaciones diplomáticas; y bajo este punto de vista dirigiré mi conducta si se tocare la materia porestegobierno, absteniéndomedehacerloyo por mi parte, porque creo que al imperio en nada le perjudica que las cosas sobre limites permanezcan en el estado de indecisión que hoy se hallan.

Esto supuesto, arreglaré mi conducta hasta el 4 de marzo a manifestar siempre queseofrezcalasdisposicionesamistosasdel imperioparaconelgobiemodeestos Estados y, sóloen el caso de ser invitadoen alguno de estos particulares que llevo 
expresados, entraré en contestaciones con mucha circunspección y detenida reflexión, persuadido de que no es llegado todavía el momento de sacar ventajas para el imperio por vía de tratados con este gobiemo.

Dije que hasta el día 4 de marzosería ésta mi conducta, porque hasta esa fecha es cuando aquí se puede tratar algo, pues desde entonces que se disuelve el Congreso todos los funcionarios del gobiemo, comenzando desde el presidente y los ministros extranjeros, se van a vivir privadamente a casas de campooal lugar que quieren de estos Estados, sin volverse a reunir hasta el primer lunes de diciembre del año inmediato, y así es que todo el tiempo que media desde 4 de marzo hasta principios de diciembre es absolutamente nulo para emprender negociaciones diplomáticas.

He dado cuenta de mis operaciones y conducta que me propongo observar. Vuestra excelencia en vista de todo me prevendrá lo que tenga por conveniente.

Dios guarde a vuestra excelencia muchos años. Washington, 26 de diciembre de 1822. Zozaya. Excelentísimo señor secretario de Estado y del Despacho de Relaciones Interiores y Exteriores del imperio?

\section{Excelentísimo señor:}

Entrelosencargosque principalmentetrajea micuidadofueunodeelloseldeabrir un préstamo hasta la cantidad de 10000000 , y aunque vuestra excelencia no me lo hubiera recomendado tan eficazmente, me bastaría para estar penetrado de la urgencia de esta medida el conocimiento que tengo de la situación actual del imperio y de la imperiosa necesidad en que se halla de mi pronto auxilio de numerario; pero a pesar de eso no me he movido a dar paso alguno sobre este particular, niauna indicarnuestra situación, lo primero porque el préstamoabierto por el ministro de Colombia en Londres, de que hablan las dos copias que acompaño a vuestra excelencia traducidas de unos manuscritos que he habido a las manos, ha desconceptuado completamente en este pais esta clase de negociaciones entabladas con gobiemos nacientes, porque se dice que el de Colombia hadesaprobadolaconductadesu ministroy nohaqueridoreconocerlosempeños que éste contrajo. Lo segundo, y principalmente, que no es este el país donde pueda entablarse negocio alguno de qué sacar numerario, porque no lo tienen ni para sí. Se manejan casi al crédito y, para confirmar este concepto, no hay más que volver la vista a la multituddebancosestablecidosen los más delos lugaresdeestos Estados; de manera que aquí casi no se conoce la moneda y todo el tráficose hace con papel.

\footnotetext{
? SRE, AEMEU, t. 424 , núm. 6 , ff. 10v-12.
} 
No he tenido el tiempo suficiente ni los datos necesarios para calcular el numerario en circulación, pero por lo que he observado hasta ahora creo muy racional el que hace Onísensu memoria de 20000000 , y siendoasí ¿cómose podrá aguardar ni remotamente el préstamo de 10000000 ?

Convencido de la imposibilidad de realizar el proyecto no he querido ni aun indicar que el imperio se halla en necesidad de numerario, porque las urgencias deben sólo manifestarse a quien pueda remediarlas.

Endefectode préstamopodía acasorecurrirseal establecimientoen el imperio deunoomásbancos, losquehan probadotanbienenestosEstadossiendodesuyo bien pobres; y por lo que pueda conducir a ilustrar este pensamiento remito un reglamento de banco en el concepto de que todos los de estos Estados están montados casi bajo de un pie con pequeñas variaciones accidentales.

Dios guarde a vuestra excelencia muchos años. Washington, 26 de diciembre de 1822. Zozaya. Excelentísimo señor secretario de Estado y del Despacho de Relaciones Interiores y Exteriores del imperio. ${ }^{8}$

\section{Exœelentísimo señor:}

La soberbia de estos republicanos no les permite vernos como iguales sino como inferiores; su envanecimiento se extiende en mi juicio a creer que su capital lo será de todas las Américas: aman entrañablemente a nuestro dinero, no a nosotros, ni son capaces de entrar en convenio de alianza o comercio sino por su propia conveniencia, desconociendo la recíproca. Con el tiempo han de ser nuestros enemigos jurados, y con tal previsión los debemos tratar desde hoy, que se nos venden amigos, de cuyo modo debemos conducimos oficial y privadamente, y consecuente a este principio o si no por meade [sid] para aprovecharnos de sus conocimientos, relaciones e influjo para el establecimiento de consulados y después poner uno de nuestra patria en todos los papeles. [ipuertos?] En las sesiones del Congreso general y en las sesiones de los estados particulares, no se habla de otra cosa que de arreglo de ejército y milicias y esto no tiene sin duda otro objeto que el de miras ambiciosas sobre la provincia de Texas. El ejército, que no pasade 10000 hombres nibajade 6000 , repartidoenlasfronterasy particularmente en la nuestra, pueden servir para defender su suelo, perono son temiblesfuera de él. Washington, 26 de diciembre de 1822. Duplicado en 2 de enero de 1823. Señalado con una núbrica.?

${ }^{8}$ SRE, AEMEU, t. 424, núm. 7, ff. 12-12v.

${ }^{9}$ Diplomacia, 1910, t. 1, p. 103. 